\title{
IRON OVERLOAD IN VERY LOW BIRTH WEIGHT INFANTS: SERUM FERRITIN AND ADVERSE OUTCOMES
}

\author{
M. Barrett ${ }^{1}$, B. Freyne ${ }^{1}$, E. Molloy ${ }^{1,2,3}$ \\ ${ }^{l}$ Neonatology, NMH, ${ }^{2}$ Medicine \& Medical Sciences, UCD, ${ }^{3}$ Paediatrics, Royal College of Surgeons, Dublin, \\ Ireland
}

Adequate iron isessential for growth and haematpoiesis. Oral iron supplementation is the standard of care in VLBW infants. Post mortem evidence has confirmed significant iron overload. Excessive free iron has been associated with free radical formation and brain injury in term infants.

Iron indices were measured in VLBW infants (weight $<1500 \mathrm{~g}$ and gestation $<32$ weeks) prior to initiation of iron. Exclusion criteria were transfusion in the preceding three days, evidence of sepsis or ventilator dependency.

45 infants were enrolled over a six month period. Ferritin $>300$ microg $/ \mathrm{L}$ was considered elevated. Data was analysed using SPSS Version 14.0. Associations were assessed using Mann Whitney U tests for nonparametric variables and $\mathrm{p}<0.05$.

Ferritin levels were elevated in $31 \%(n=14)$. The mean ferritin level was 270 microgms $/ \mathrm{L}$. The mean total Iron was within normal limits at 16.64umol (11-34umol). However mean Transferrin and TIBC levels were below normal at $1.75 \mathrm{~g} / \mathrm{dl}(2-3 \mathrm{~g} / \mathrm{dl})$ and $42.35 \mathrm{umol}$ (50-85umol) respectively. 19 MRIs had been conducted at term. Of 6 MRIs conducted in the high ferritin group, 3 were abnormal and 3 were normal. In the low ferritin group 13 MRIS were conducted at term, 10 were normal and 3 were abnormal.

In this group of healthy VLBW infants there was a $31 \%$ of iron overload at time of starting routine iron supplementation. ELBW and lower gestational age were risk factors for developing iron overload which seems to be associated with number of transfusions required. VLBW infants with high ferritin longer on both NCPAP and 02. 